\title{
Neural variability, or lack thereof
}

\section{Timothée Masquelier ${ }^{1,2 * t}$}

${ }^{1}$ Unit for Brain and Cognition, Department of Information and Communication Technologies, Universitat Pompeu Fabra, Barcelona, Spain

2 Laboratory of Neurobiology of Adaptive Processes, UMR 7102, CNRS - University Pierre and Marie Curie, Paris, France

\section{Edited by:}

Klaus R. Pawelzik, University

Bremen, Germany

Reviewed by:

Thomas Boraud, Universite de

Bordeaux, France

\section{${ }^{*}$ Correspondence:}

Timothée Masquelier, Unit for Brain and Cognition, Universitat Pompeu

Fabra, Barcelona, Spain.

e-mail: timothee.masquelier@

alum.mit.edu

${ }^{\dagger}$ Present address:

Timothée Masquelier, Adaptive

NeuroComputation Group,

Laboratory of Neurobiology of

Adaptive Processes, UMR 7102,

CNRS - University Pierre and Marie

Curie, Box 12, Building B, 5th Floor,

Room 519B, 9 Quai St. Bernard,

75005 Paris, France.
We do not claim that the brain is completely deterministic, and we agree that noise may be beneficial in some cases. But we suggest that neuronal variability may be often overestimated, due to uncontrolled internal variables, and/or the use of inappropriate reference times. These ideas are not new, but should be re-examined in the light of recent experimental findings: trial-to-trial variability is often correlated across neurons, across trials, greater for higher-order neurons, and reduced by attention, suggesting that "intrinsic" sources of noise can only account for a minimal part of it. While it is obviously difficult to control for all internal variables, the problem of reference time can be largely avoided by recording multiple neurons at the same time, and looking at statistical structures in relative latencies. These relative latencies have another major advantage: they are insensitive to the variability that is shared across neurons, which is often a significant part of the total variability. Thus, we suggest that signal-to-noise ratios in the brain may be much higher than usually thought, leading to reactive systems, economic in terms of number of neurons, and energy efficient.

\section{Keywords: neural variability, signal-to-noise ratio, reliability, redundancy, neural coding}

\section{INTRODUCTION}

Randomness is only a measure of our "ignorance of the different causes involved in the production of events" (Laplace, 1825).

High trial-to-trial variability in response to repeated presentation of a same stimulus has been reported in every modality. It is often quantified in terms of reliability and precision (Box 1), and both are usually poor in vivo (e.g., Fano factors $\sim 1$ and precision $\sim$ tens of $\mathrm{ms}$ or above). The origin of this variability, and its implication for information processing, has been much debated (Stein et al., 2005; Ermentrout et al., 2008; Faisal et al., 2008; Tiesinga et al., 2008; Rolls and Deco, 2010), yet a consensus has not emerged. Here we argue that most of the observed variability could come from uncontrolled variables, or the use of inappropriate reference times, rather than from intrinsic sources of noise ("intrinsic" meaning that they cannot be eliminated). We focus on sensory systems, where signals are best identified, yet in general not perfectly.

\section{THE FUNCTIONAL APPROACH: NOISE, OR UNCONTROLLED VARIABLES?}

Noise is a relative concept. It measures the extent to which a system diverges from its hypothesized, idealized, function. For example neurons in early sensory areas are usually hypothesized to encode stimulus features, and only stimulus features. Then trial-to-trial variability in their activity, when controlling for the stimulus, may be called "noise." If this variability is lower than the variability between different stimuli, then the hypothesis is validated a posteriori. Similarly, neurons in primary motor areas are hypothesized to encode motor responses (and only motor responses), and the variability across trials with the same motor response is "noise."

Unfortunately, neurons' functions are generally unknown, in particular for "higher-order neurons" (that is, farther away from sensory inputs and motor outputs), hence the term "noise" should be used with caution, and the term "unexplained variability" should be preferred. Furthermore, a neuron's function may change over time. For example V1 neurons, when the eyes are closed or in the dark, can be involved in mental imagery (Kosslyn and Thompson, 2003). An experimenter unaware of this will observe a huge unexplained variability in neural activity if he/she fails to control for mental imagery - which is of course difficult and will lead to some variability anyway.

In this paper, we argue that most of the unexplained variability in sensory systems might result from deterministic, but uncontrolled, internal variables mediating attention, degree of arousal, expectations, mental imagery, task-solving strategies, etc. This variability is signal, even though it would look like noise to an experimenter only controlling for the stimulus-all the more so because we know from Shannon's theory of information that when optimal encoding is used to maximize information transmission, neural signals will look random (Faisal et al., 2008). As Barlow wrote about neural responses in 1972, "their apparently erratic behavior was caused by our ignorance, not the neuron's incompetence" (Barlow, 1972).

An extreme case occurs when there are no external variables at all, only (uncontrolled) internal ones, that is when recording spontaneous activity. It should not come as a surprise that trial-to-trial variability is higher in this case than when a same stimulus is repeated, as seen in a number of experiments (Churchland et al., 2010). Indeed, why should activity be 
the same across trials in which nothing repeats? In some sense, it would be "fairer" to compare spontaneous activity's variability to evoked activity's without controlling for the stimulus that is using varied stimuli. Yet of course this approach has flaws too: the results would dependent on how varied the stimuli are.

Since it has a metabolic cost, the spontaneous activity probably has a function. The observed variability might reflect more our inability to grasp it, and to control for appropriate variables, than neurons' unreliability.

\section{THE BIOLOGICAL APPROACH: INTRINSIC AND EXTRINSIC SOURCES OF VARIABILITY}

Coming back to evoked responses, what mechanisms may cause the commonly observed high trial-to-trial variability? In vitro, single neurons stimulated directly by injecting fluctuating currents, in the absence of synaptic input, give highly reliable and (sub)millisecond precise responses (Bryant and Segundo, 1976; Mainen and Sejnowski, 1995; Toups et al., 2012), that deterministic neuronal models can accurately predict (Gerstner and Naud, 2009), despite "channel noise" (Faisal et al., 2008). In a sensory neuronal network, there are two additional intrinsic sources of variability: sensors, which convert physical stimuli into spikes, and synaptic transmission. In many cases, sensors operate close to physical limits that introduce variability (Stein et al., 2005), and therefore should contribute minimally to the variability observed in vivo. Synaptic unreliability may have a bigger impact (Movshon, 2000; Faisal et al., 2008). However, high reliability and (sub)millisecond precision is seen in cortex in some experiments (see Tiesinga et al., 2008; Haider et al., 2010; Kayser et al., 2010; Panzeri et al., 2010; Herikstad et al., 2011, and references therein), suggesting that it is possible for the brain to overcome this source of variability (Mainen and Sejnowski, 1995), most probably because it is largely independent across synapses, and thus averaged out when a neuron integrates from many of them (we will come back to this point).

So why is trial-to-trial variability so high in other experiments? It could be because: (a) neurons' states when presenting the stimulus differ; (b) neurons receive, in addition to controlled bottom-up sensory signals, uncontrolled top-down extrasensory signals. Both of these variability sources are called "extrinsic," because in principle they could be eliminated by proper control. We call (c) the intrinsic sources of variability reviewed above (sensors, ion channels, and synapses). In the following sections, we try to rule in or out each possible source of variability in the light of recent experimental findings.

\section{INTER-NEURON CORRELATIONS}

Trial-to-trial variability is typically correlated across neurons (Averbeck et al., 2006), a phenomenon sometimes called "correlated noise." In other words, a significant part of the total variability is often shared across neurons (Churchland et al., 2010). Variability caused by (c) is expected to be largely independent across neuron. (c) is thus largely ruled out (Table 1, first line).

Variability caused by (a) can be correlated across neurons if a common signal determines the neurons' states at stimulus onset. There is much evidence for such signals, which are typically oscillating, and the phase at which a stimulus is presented modulates both evoked neural responses and behavioral performance, in the visual (Vanrullen et al., 2011), auditory (Ng et al., 2012), and somatosensory (Palva et al., 2005) modalities. Therefore (a) is ruled in. Besides, it is worth mentioning that spikes may to lock to these internal oscillations, not to the stimulus onset (Izhikevich, 2006; Tiesinga et al., 2008; Masquelier et al., 2009b; Panzeri et al., 2010). Therefore, using the stimulus onset as a reference time when computing Fano factors or spike time dispersion is inappropriate: it would lead to high values that do not reflect the real reliability and precision (Figure 1).

Variability caused by (b) will be often correlated across neurons as well: top-down signals will typically target multiple neurons and influence similarly their activity. For example in the visual system spatial attention will target all neurons whose receptive fields are in the attended region. Feature-based attention will target all neurons coding for a same feature. Top-down signal are also hypothesized to encore prior expectations, used in a Bayesian inference process (see Chikkerur et al., 2010, and references therein), which will also tend to be similar for neurons coding for similar features. Mental imagery will typically activate similarly all neurons representing the imagined thing. So (b) is ruled in.

Finally, are these inter-neurons correlations beneficial, or detrimental? They are often seen as detrimental, because only independent noise can be efficiently averaged out in a population coding framework, that is when a signal's magnitude is estimated by averaging responses across a pool of neurons with similar tuning properties (Averbeck et al., 2006; Cohen and Kohn, 2011), possibly thanks to stochastic resonance (Stein et al., 2005; Faisal

Table 1 | Ruling in and ruling out variability sources (a) different states at stimulus onset, (b) top-down extrasensory signals, (c) intrinsic (sensors, ion channels, and synapses).

\begin{tabular}{ll}
\hline Experimental finding & $\begin{array}{l}\text { Implications for main variability } \\
\text { sources }\end{array}$ \\
\hline Inter-neuron correlations & (a) (b) (c) \\
Inter-trial correlations & (a, but only for infraslow oscillations) \\
Greater variability for & (b) (c) \\
higher-order neurons & (b) (e, at least for rapid, feedforward \\
Attention quenches variability & processing) \\
\end{tabular}



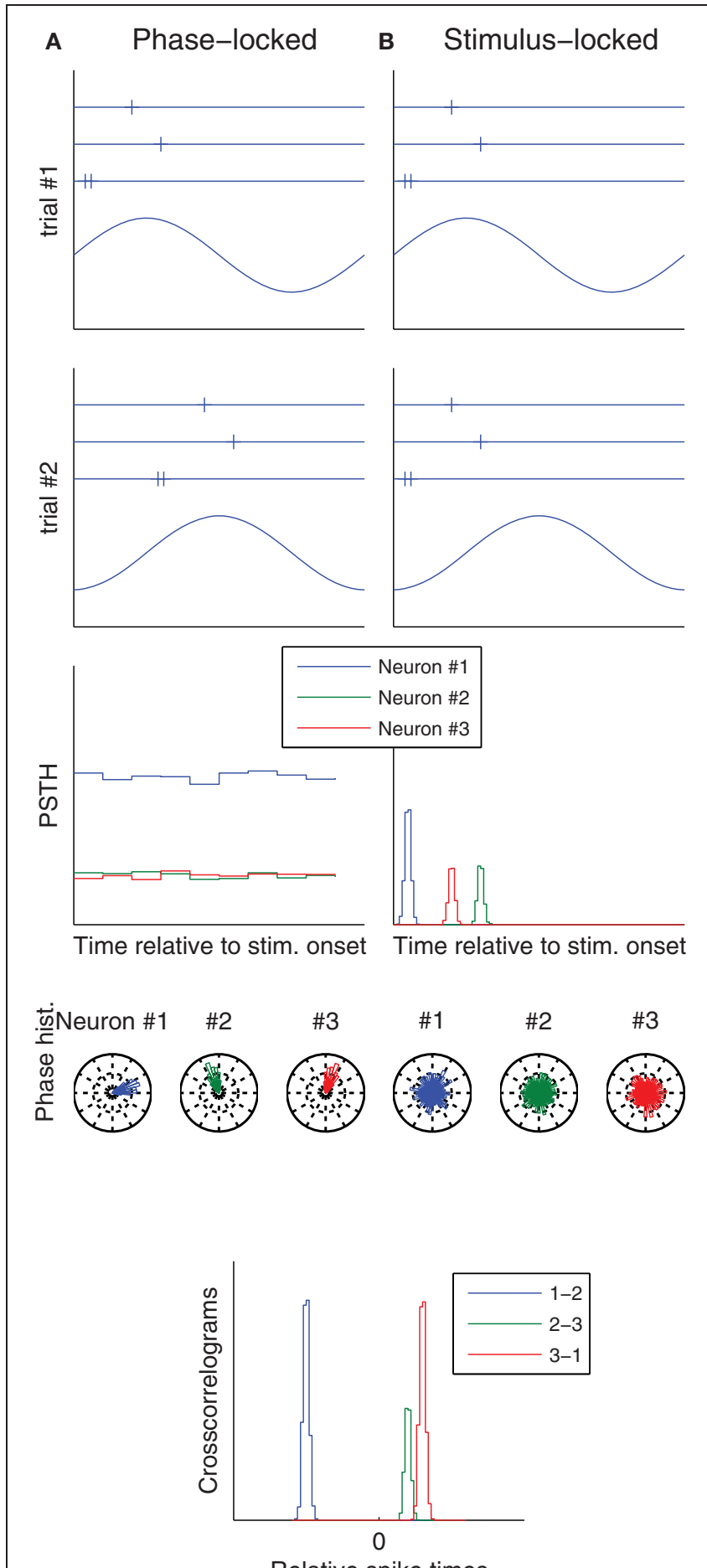

Relative spike times

FIGURE 1 | Phase vs. stimulus locking. Column (A) (resp. B) illustrates a situation in which spikes lock to an ongoing oscillation (resp. to stimulus onset). The first two rows correspond to two trials, and show both the raster plots of three neurons (top), and the ongoing oscillation (bottom), whose phase at stimulus onset is different from trial-to-trial. Post-stimulus time histograms (PSTH), which use the stimulus onset as a reference time, only reveal the temporal structure in the stimulus locked-case. Conversely, spike phase histograms, which use the oscillation peak as a reference time, only reveal the temporal structure in the phase locked-case.

(Continued)

\section{FIGURE 1 | Continued}

Spike time cross-correlograms between pairs of neurons reveal the temporal structure in both cases. These are good news, because downstream neurons only care about relative spike times-they ignore both the stimulus onset time and the oscillation phase.

et al., 2008) (Box 2). However, we are notoriously imprecise at "absolute" level estimations if stimuli are presented one at a time (Miller, 1956). We are much better at comparing simultaneously presented stimuli (Stewart et al., 2005). It is likely that we do so by comparing different neurons' activities. In this case, interneurons correlations are not detrimental, and even preferable to independent variability: from trial-to-trial, activities will tend to be all shifted in the same direction, preserving the order, thus the relative judgment.

More specifically, mean spike counts (or latencies), averaged across neurons, could depend on neurons' states at stimulus onset [source (a)], and/or on top-down signals [source (b)], and thus show trial-to-trial variability, while relative spike counts (or latencies) could robustly encode stimulus features (Figure 2). So once again, the observed variability might reflect a wrong assumption of us scientists (stimuli are encoded in absolute spike counts/latencies), more than neurons' unreliability. In line with this proposition, relative latencies have been found to encode stimuli more robustly than absolute ones, in the visual (Desbordes et al., 2008; Gollisch and Meister, 2008; Havenith et al., 2011; Masquelier, 2012; Shriki et al., 2012), somatosensory (Johansson and Flanagan, 2009; Panzeri and Diamond, 2010), auditory (Chase and Young, 2007; Brasselet et al., 2012), and olfactory (Junek et al., 2010; Schaefer and Margrie, 2012) systems.

\section{INTER-TRIAL CORRELATIONS}

Trial-to-trial variability is often correlated over extended timescales, of tens of seconds or above, which typically involve multiple trials (Monto et al., 2008; Marom, 2010; Marom and Wallach, 2011). What does that tell us about the possible variability sources?

These inter-trial correlations could be caused again by an ongoing oscillation, provided its period is longer than intertrial intervals (typically a few seconds). There is evidence for such "infraslow" $(0.01-0.1 \mathrm{~Hz})$ ongoing EEG oscillations in the somatosensory system, whose phase predicts detection performance (Monto et al., 2008). Therefore (a) is ruled in, but only for infraslow oscillations.

In addition, when an organism is not passively sensing but has to solve a task, these long timescales could be the signature of high-level meta-cognitive processes, in charge of implementing different task-solving strategies, presumably thanks to top-down signals, for example subject-object coupled dynamic exploration (Marom and Wallach, 2011), and changes of these strategies, for example shifting the speed-accuracy or aggressive-conservator tradeoffs, or starting paying more attention to some diagnostic features. Therefore (b) is ruled in.

The mechanisms causing intrinsic variability (sensors, ion channels, and synapses) are commonly thought to have short timescales $(<<s)$. Even though subtle longer term memory effects are sometimes seen (Marom, 2010), they could only 


\section{Box 2 | Stochastic resonance.}

In neuronal networks, subthreshold signals are not transmitted-only spikes are. This somehow binarizes signals, which can be either subthreshold (no spike), or suprathreshold (spikes). In principle, the magnitude of a suprathreshold signal can be estimated by averaging the firing rate across a long time window. But in practice, it is often not possible because a decision has to be taken rapidly. In any case, it seems that in the brain, neurons' inputs are subthreshold most of the time (Abeles, 1982; König et al., 1996; Brette, 2012). Hence being able to transmit subthreshold signals would greatly enhance the bandwidth. With this goal in mind, it has been suggested that adding noise to a subthreshold signal will cause occasional firings, and more often for near-threshold signals. Therefore, the original subthreshold signal can be estimated by averaging firing rates across time, or across neurons receiving the same subthreshod signal (again, this second option, referred to as "population coding," is more realistic when reactivity is an issue). Importantly, to be efficiently averaged out, the noise has to be independent across neurons. Besides, there is an optimal level of noise: if too weak, the threshold is not reached often enough; if too strong, the response is dominated by the noise; hence the term "stochastic resonance."

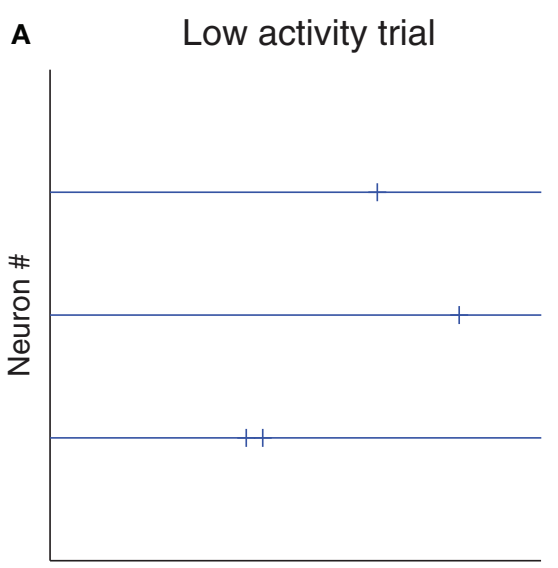

B

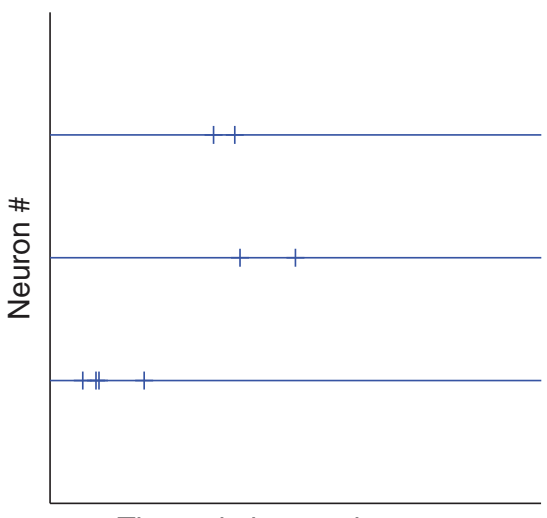

Time relative to stim. onset

FIGURE 2 | Shared variability is not detrimental to relative coding schemes. Here we illustrate a hypothetical situation in which most of the trial-to-trial variability is shared. (A) Raster plot of a trial with low spike counts and/or long latencies (for example because the stimulus was presented at a suboptimal phase [source (a)], or because the subject was not attentive [source (b)]. (B) Trial with high spike counts and/or short latencies (for the opposite reasons). If these two kinds of trials are observed, Fano factors and spike time dispersion will be high. However, relative spike counts and/or latencies could be more reproducible (because both sources (a) and (b) could affect spike counts and/or latencies similarly across neurons), and could robustly encode the stimulus. Of course, detecting such cases of "relative coding" requires recording multiple neurons at a time, and looking at stimulus-dependent statistical structure in the cross-correlograms. Conversely, neither a PSTH nor a phase histogram (Figure 1) would help. account for a very small part of inter-trial correlations. Therefore (c) is largely ruled out.

\section{GREATER VARIABILITY FOR HIGHER-ORDER NEURONS}

Variability is typically greater for higher-order neurons. For example in the visual system, both reliability and precision tend to decrease along the ventral pathway (Tiesinga et al., 2008; Herikstad et al., 2011), while top-down effects are greater and greater (Buffalo et al., 2010). Variability is minimal in the retina (Kara et al., 2000; Movshon, 2000), which is out of reach of top-down signals. This is consistent with the proposition that top-down effects are responsible for most of the neural variability. (b) is ruled in. Furthermore, it seems that it is essentially the shared variability, not so much the private one, which increases along the hierarchy: noise correlations are typically low in V1 (Ecker et al., 2010), and higher in extrastriate areas (Faisal et al., 2008). This is again consistent with (b) being a major source of (shared) variability.

To say the same thing in functional terms: higher-order neurons may appear more variable because in general we know less what they are signaling, which may not only be related to the physical stimulus. When by chance we happen to know what a higher-order neuron is signaling, for example a person's identity in case of a so called "grand-mother cell," or "concept cell," which selectively responds to photographs of the person as well as his/her written name, then variability is in fact low enough so that this identity can be robustly readout from this sole neuron in a single trial (Quiroga et al., 2005).

How about source (c)? It is estimated that the equivalent of about 50 synchronous excitatory postsynaptic potentials (EPSPs) are required to elicit a postsynaptic spike (Sherwood, 2012). Note that this does not imply redundancy: the 50 presynaptic neurons may be signaling different features, and postsynaptic spikes signal the conjunction of them. But it does imply that independent variability in EPSPs, such as the one caused by (c), will be largely averaged out, and postsynaptic spikes will tend to be more reliable and precise than presynaptic ones. Consequently, spike patterns can be reliably transmitted in feedforward networks, without jitter accumulation (Kumar et al., 2010). This is not consistent with higher-order neurons being more variable, thus (c) is largely ruled out, at least when processing is massively feedforward, that is for rapid sensory processing. When reactivity is less of an issue, the brain can accumulate evidence through recurrent processing (Masquelier et al., 2011). Feedback connectivity makes networks 
chaotic (Izhikevich and Edelman, 2008; London et al., 2010), that is highly sensitive to small perturbations such as the ones caused by (c), which will end up impacting the whole network. It is thus unclear if higher-order neurons should or should not be more variable in that case, meaning that (c) is neither ruled in, nor ruled out.

It is also unclear if variability caused by different neuronal states at stimulus onset would be greater or lower for higher-order neurons, therefore (a) is neither ruled in, nor ruled out.

\section{ATTENTION QUENCHES VARIABILITY}

Attention can reduce response (shared) variability (Mitchell et al., 2007, 2009; Cohen and Maunsell, 2009). This rules out a big role for intrinsic sources of noise (c), whereas both sources (a) and (b) are ruled in. The decrease in response variability could be due to some active mechanisms quenching pre-stimulus activity variability, especially when the time-point of the stimulus is predictable (Ledberg et al., 2012), suggesting that (a) might be the main source of variability. Importantly, these drops in variability of both spontaneous and evoked activity lead to improved behavioral performance (Ledberg et al., 2012). Thus, it seems that neural variability is globally detrimental to sensory processing, and that the brain tries to limit it through active mechanisms (we will come back to this point).

\section{CONCLUSIONS}

In some cases neural responses are both reliable precise, even in cortex (see Tiesinga et al., 2008; Haider et al., 2010; Kayser et al., 2010; Panzeri et al., 2010; Herikstad et al., 2011, and references therein). This suggests that when it is not the case, it might not be because of intrinsic sources of noise, but rather because (1) We did not understand the neuron's function, and thus failed to control for appropriate variables and/or (2) We used an inappropriate reference time, for example the stimulus onset, while spikes locked to an internal ongoing oscillation, or vice-versa (Figure 1). In accordance with these two suggestions, the variability is typically correlated across neurons, across trials, greater for higher-order neurons, and quenched by attention (Table 1).

While it is obviously difficult to control for all extrasensory variables, the problem of reference time can be largely avoided by recording neurons simultaneously, and looking at relative spike time statistical structure using cross-correlograms. Relative latencies have another advantage: they are insensitive to the variability shared across neurons (Figure 2), and are thus often less

\section{REFERENCES}

Abeles, M. (1982). Role of the cortical neuron: integrator or coincidence detector? Isr. J. Med. Sci. 18, 83-92.

Amarasingham, A., Chen, T., Geman, S., Harrison, M., and Sheinberg, D. (2006). Spike count reliability and the poisson hypothesis. J. Neurosci. 26, 801-809.

Averbeck, B. B., Latham, P. E., and Pouget, A. (2006). Neural

variable than absolute ones (Chase and Young, 2007; Desbordes et al., 2008; Gollisch and Meister, 2008; Johansson and Flanagan, 2009; Junek et al., 2010; Panzeri and Diamond, 2010; Havenith et al., 2011; Brasselet et al., 2012; Masquelier, 2012; Schaefer and Margrie, 2012; Shriki et al., 2012). These are good news, because downstream neurons only care about relative latencies, and the required connectivity to decode them can spontaneously emerge with spike timing-dependent plasticity (Masquelier et al., 2008, 2009a; Gilson et al., 2011; Brette, 2012).

Neurons may thus be more reliable and precise than usually thought, allowing lower redundancy in the brain, that is fewer neurons for a same level of robustness, which is obviously desirable, but also fewer spikes, and thus lower metabolic costs. In line with this proposal, it has been shown that stimulating very few cortical neurons, sometimes only one, and generating only a few extra spikes, can impact behavior (Wolfe et al., 2010). Variability, and thus redundancy, could be particularly low when dealing with suprathreshold stimuli (Gur and Snodderly, 2006) - a more natural situation - and with natural rather than artificial stimuli (Haider et al., 2010; Hasson et al., 2010; Herikstad et al., 2011).

Of course, population coding with redundant noisy neurons would have other theoretical advantages. We have already mentioned stochastic resonance (Box 2). In addition, neuron populations could encode probability distribution over the stimulus, and not only most probable values, and combine them optimally, provided the noise is Poisson-like (Ma et al., 2006). For long processing times (say hundreds of milliseconds or above), noise, or, more accurately, fluctuations, have other benefits: they allow not getting stuck in deadlocks, in a local minima in a minimization problem, or exploring various attractors in multistable perception (Martí et al., 2008; Rolls and Deco, 2010). Importantly, these fluctuations could come from chaotic reverberating activity (Izhikevich and Edelman, 2008; London et al., 2010), exacerbating potentially very weak intrinsic noise. Consistent with this idea, the beginning of responses-which is mostly shaped by feedforward inputs-is typically less variable than the rest of it—shaped by feedback as well (Amarasingham et al., 2006; Churchland et al., 2010).

But despite these potential benefits, we feel that it is too early to conclude that stochasticity is ubiquitous in the brain, and always essential to its function. In a number of cases, it seems to be a nuisance, that can be reduced by attention (Ledberg et al., 2012) and training (Qi and Constantinidis, 2012; Verstynen et al., 2012) both might in fact shift the neuron's functions towards ones we understand better, leading to the apparent variability reduction.

primate auditory cortex. J. Neurosci. 32, 2998-3008.

correlations, population coding and computation. Nat. Rev. Neurosci. 7 , 358-366.

Barlow, H. B. (1972). Single units and sensation: a neuron doctrine for perceptual psychology? Perception 1 , 371-394.

Brasselet, R., Panzeri, S., Logothetis, N. K., and Kayser, C. (2012). Neurons with stereotyped and rapid responses provide a reference frame for relative temporal coding in
Brette, R. (2012). Computing with neural synchrony. PLoS Comput. Biol. 8:e1002561. doi: 10.1371/journal.pcbi.1002561

Bryant, H. L., and Segundo, J. P. (1976). Spike initiation by transmembrane current: a white-noise analysis. J. Physiol. 260, 279-314.

Buffalo, E. A., Fries, P., Landman, R., Liang, H., and Desimone, R. (2010).
A backward progression of attentional effects in the ventral stream. Proc. Natl. Acad. Sci. U.S.A. 107, 361-365.

Chase, S. M., and Young, E. D. (2007). First-spike latency information in single neurons increases when referenced to population onset. Proc. Natl. Acad. Sci. U.S.A. 104, 5175-5180.

Chikkerur, S., Serre, T., Tan, C., and Poggio, T. (2010). What and 
where: a bayesian inference theory of attention. Vision Res. 50, 2233-2247.

Churchland, M. M., Yu, B. M., Cunningham, J. P., Sugrue, L. P., Cohen, M. R., Corrado, G. S., et al. (2010). Stimulus onset quenches neural variability: a widespread cortical phenomenon. Nat. Neurosci. 13, 369-378

Cohen, M. R., and Kohn, A. (2011). Measuring and interpreting neuronal correlations. Nat. Neurosci. 14, 811-819.

Cohen, M. R., and Maunsell, J. H. R. (2009). Attention improves performance primarily by reducing interneuronal correlations. Nat. Neurosci. 12, 1594-1600.

Desbordes, G., Jin, J., Weng, C., Lesica, N. A., Stanley, G. B., and Alonso, J.-M. (2008). Timing precision in population coding of natural scenes in the early visual system. PLoS Biol. 6:e324. doi: 10.1371/journal.pbio.0060324

Ecker, A. S., Berens, P., Keliris, G. A., Bethge, M., Logothetis, N. K., and Tolias, A. S. (2010). Decorrelated neuronal firing in cortical microcircuits. Science 327, 584-587.

Ermentrout, G. B., Galán, R. F., and Urban, N. N. (2008). Reliability, synchrony and noise. Trends Neurosci. 31, 428-434.

Faisal, A. A., Selen, L. P. J., and Wolpert, D. M. (2008). Noise in the nervous system. Nat. Rev. Neurosci. 9, 292-303.

Gerstner, W., and Naud, R. (2009). How good are neuron models? Science 326, 379-380.

Gilson, M., Masquelier, T., and Hugues, E. (2011). STDP allows fast ratemodulated coding with poissonlike spike trains. PLoS Comput. Biol. 7:e1002231. doi: 10.1371/journal.pcbi.100223

Gollisch, T., and Meister, M. (2008). Rapid neural coding in the retina with relative spike latencies. Science 319, 1108-1111.

Gur, M., and Snodderly, D. M. (2006). High response reliability of neurons in primary visual cortex (v1) of alert, trained monkeys. Cereb. Cortex 16, 888-895.

Haider, B., Krause, M. R., Duque, A., Yu, Y. G., Touryan, J., Mazer, J. A., et al. (2010). Synaptic and network mechanisms of sparse and reliable visual cortical activity during nonclassical receptive field stimulation. Neuron 65, 107-121.

Hasson, U., Malach, R., and Heeger, D. J. (2010). Reliability of cortical activity during natural stimulation. Trends Cogn. Sci. 14, 40-48.
Havenith, M. N., Yu, S., Biederlack, J., Chen, N.-H., Singer, W., and Nikolic, D. (2011). Synchrony makes neurons fire in sequence, and stimulus properties determine who is ahead. J. Neurosci. 31, 8570-8584.

Herikstad, R., Baker, J., Lachaux, J.-P., Gray, C. M., and Yen, S.-C. (2011). Natural movies evoke spike trains with low spike time variability in cat primary visual cortex. J. Neurosci. 31, 15844-15860.

Izhikevich, E. M. (2006). Polychronization: computation with spikes. Neural Comput. 18, 245-282.

Izhikevich, E. M., and Edelman, G. M. (2008). Large-scale model of mammalian thalamocortical systems. Proc. Natl. Acad. Sci. U.S.A. 105, 3593-3598.

Johansson, R. S., and Flanagan, J. R. (2009). Coding and use of tactile signals from the fingertips in object manipulation tasks. Nat. Rev. Neurosci. 10, 345-359.

Junek, S., Kludt, E., Wolf, F., and Schild, D. (2010). Olfactory coding with patterns of response latencies. Neuron 67, 872-884.

Kara, P., Reinagel, P., and Reid, R. C. (2000). Low response variability in simultaneously recorded retinal, thalamic, and cortical neurons. Neuron 27, 635-646.

Kayser, C., Logothetis, N. K., and Panzeri, S. (2010). Millisecond encoding precision of auditory cortex neurons. Proc. Natl. Acad. Sci. U.S.A. 107, 16976-16981.

König, P., Engel, A. K., and Singer, W. (1996). Integrator or coincidence detector? the role of the cortical neuron revisited. Trends Neurosci. 19, 130-137.

Kosslyn, S. M., and Thompson, W. L. (2003). When is early visual cortex activated during visual mental imagery? Psychol. Bull. 129, 723-746.

Kumar, A., Rotter, S., and Aertsen, A. (2010). Spiking activity propagation in neuronal networks: reconciling different perspectives on neural coding. Nat. Rev. Neurosci. 11, 615-627.

Laplace, P. (1825). Essai Philosophique sur les Probabilités. Paris: GauthierVillars.

Ledberg, A., Montagnini, A., Coppola, R., and Bressler, S. L. (2012). Reduced variability of ongoing and evoked cortical activity leads to improved behavioral performance. PLoS ONE 7:e43166. doi: 10.1371/journal.pone.0043166
London, M., Roth, A., Beeren, L. Häusser, M., and Latham, P. E. (2010). Sensitivity to perturbations in vivo implies high noise and suggests rate coding in cortex. Nature 466, 123-127.

Ma, W. J., Beck, J. M., Latham, P. E., and Pouget, A. (2006). Bayesian inference with probabilistic population codes. Nat. Neurosci. 9 1432-1438.

Mainen, Z. F., and Sejnowski, T. J. (1995). Reliability of spike timing in neocortical neurons. Science 268 , 1503-1506.

Marom, S. (2010). Neural timescales or lack thereof. Prog. Neurobiol. 90, 16-28.

Marom, S., and Wallach, A. (2011) Relational dynamics in perception: Impacts on trial-to-trial variation. Front. Comput. Neurosci. 5:16. doi: 10.3389/fncom.2011.00016

Martí, D., Deco, G., Mattia, M. Gigante, G., and Giudice, P. D (2008). A fluctuation-driven mechanism for slow decision processes in reverberant networks. PLoS ONE 3:e2534. doi: 10.1371/journal.pone.0002534

Masquelier, T. (2012). Relative spike time coding and STDP-based orientation selectivity in the early visual system in natural continuous and saccadic vision: a computational model. J. Comput. Neurosci. 32, 425-441.

Masquelier, T., Albantakis, L., and Deco, G. (2011). The timing of vision - how neural processing links to different temporal dynamics. Front. Psychol. 2:151. doi: 10.3389/fpsyg.2011.00151

Masquelier, T., Guyonneau, R., and Thorpe, S. J. (2008). Spike timing dependent plasticity finds the start of repeating patterns in continuous spike trains. PLoS ONE 3:e1377. doi: 10.1371/journal.pone.0001377

Masquelier, T., Guyonneau, R. and Thorpe, S. J. (2009a). Competitive STDP-based spike pattern learning. Neural Comput. 21, 1259-1276.

Masquelier, T., Hugues, E., Deco, G., and Thorpe, S. J. (2009b) Oscillations, phase-of-firing coding, and spike timing-dependent plasticity: an efficient learning scheme. J. Neurosci. 29, 13484-13493.

Miller, G. A. (1956). The magical number seven plus or minus two: some limits on our capacity for processing information. Psychol. Rev. 63, 81-97.

Mitchell, J. F., Sundberg, K. A., and Reynolds, J. H. (2007). Differential attention-dependent response modulation across cell classes in macaque visual area v4. Neuron 55 , 131-141.

Mitchell, J. F., Sundberg, K. A., and Reynolds, J. H. (2009). Spatial attention decorrelates intrinsic activity fluctuations in macaque area v4. Neuron 63, 879-888.

Monto, S., Palva, S., Voipio, J., and Palva, J. M. (2008). Very slow eeg fluctuations predict the dynamics of stimulus detection and oscillation amplitudes in humans. J. Neurosci. 28, 8268-8272.

Movshon, J. A. (2000). Reliability of neuronal responses. Neuron 27, 412-414.

Ng, B. S. W., Schroeder, T., and Kayser, C. (2012). A precluding but not ensuring role of entrained low-frequency oscillations for auditory perception. J. Neurosci. 32, 12268-12276

Palva, S., Linkenkaer-Hansen, K. Näätänen, R., and Palva, J. M. (2005). Early neural correlates of conscious somatosensory perception. J. Neurosci. 25 $5248-5258$

Panzeri, S., Brunel, N., Logothetis, N. K., and Kayser, C. (2010). Sensory neural codes using multiplexed temporal scales. Trends Neurosci. 33, 111-120.

Panzeri, S., and Diamond, M. E. (2010). Information carried by population spike times in the whisker sensory cortex can be decoded without knowledge of stimulus time. Front. Synaptic Neurosci. 2:17. doi: 10.3389/fnsyn.2010.00017

Qi, X.-L., and Constantinidis, C. (2012). Variability of prefrontal neuronal discharges before and after training in a working memory task. PLoS ONE 7:e41053. doi: 10.1371/journal.pone.0041053

Quiroga, R. Q., Reddy, L., Kreiman, G., Koch, C., and Fried, I. (2005). Invariant visual representation by single neurons in the human brain. Nature 435, 1102-1107.

Rolls, E. T., and Deco, G. (2010). The Noisy Brain. Oxford, UK: Oxford University Press.

Schaefer, A. T., and Margrie, T. W. (2012). Psychophysical properties of odor processing can be quantitatively described by relative action potential latency patterns in mitral and tufted cells. Front. Syst. Neurosci. 6:30. doi: 10.3389 /fnsys.2012.00030

Sherwood, L. (2012). Human Physiology: From Cells to Systems. Stamford, CT: Brooks/Cole Pub Co. Shriki, O., Kohn, A., and Shamir, M. (2012). Fast coding of orientation in primary visual cortex. PLoS 
Comput. Biol. 8:e1002536. doi: 10.1371/journal.pcbi.1002536

Stein, R. B., Gossen, E. R., and Jones, K. E. (2005). Neuronal variability: noise or part of the signal? Nat. Rev. Neurosci. 6, 389-397.

Stewart, N., Brown, G. D. A., and Chater, N. (2005). Absolute identification by relative judgment. Psychol. Rev. 112, 881-911.

Tiesinga, P., Fellous, J.-M., and Sejnowski, T. J. (2008). Regulation of spike timing in visual cortical circuits. Nat. Rev. Neurosci. 9, 97-107.

Toups, J. V., Fellous, J.-M., Thomas, P. J., Sejnowski, T. J., and Tiesinga,
P. H. (2012). Multiple spike time patterns occur at bifurcation points of membrane potential dynamics. PLoS Comput. Biol. 8:e1002615. doi: 10.1371/journal. pcbi. 1002615

Vanrullen, R., Busch, N. A., Drewes, J., and Dubois, J. (2011). Ongoing eeg phase as a trial-by-trial predictor of perceptual and attentional variability. Front. Psychol. 2:60. doi: 10.3389/fpsyg. 2011.00060

Verstynen, T., Phillips, J., Braun, E., Workman, B., Schunn, C., and Schneider, W. (2012). Dynamic sensorimotor planning during long-term sequence learning: the role of variability, response chunking and planning errors. PLOS ONE 7:e47336. doi: 10.1371/journal.pone.0047336

Wolfe, J., Houweling, A. R., and Brecht, M. (2010). Sparse and powerful cortical spikes. Curr. Opin. Neurobiol. 20, 306-312.

Conflict of Interest Statement: The author declares that the research was conducted in the absence of any commercial or financial relationships that could be construed as a potential conflict of interest.
Received: 06 November 2012; accepted: 03 February 2013; published online: 25 February 2013.

Citation: Masquelier T (2013) Neural variability, or lack thereof. Front. Comput. Neurosci. 7:7. doi: 10.3389/ fncom.2013.00007

Copyright (C) 2013 Masquelier. This is an open-access article distributed under the terms of the Creative Commons Attribution License, which permits use, distribution and reproduction in other forums, provided the original authors and source are credited and subject to any copyright notices concerning any third-party graphics etc. 